\title{
See You in San Francisco!
}

The Materials Research Society is identified with its meetings more than almost anything else it does. It was created to organize meetings, and it organizes some of the best meetings on materials research topics anywhere in the world. We have developed other excellent programs over the years, too, including some fine publications like this one, but it is the meetings that most clearly identify what MRS is about-timely, high-quality communication about current issues in materials research.

MRS meetings are created in a unique process that is beginning to be emulated, in part, by some of the other materials societies and by the various adhering bodies of the International Union of Materials Research Societies (IUMRS). The most distinct feature of our meetings is that no part of them is controlled by any technical standing committees. The meeting programs are created by the meeting chairs, starting with a blank sheet of paper (at least metaphorically speaking, in an increasingly paper-free age). The set of technical symposia is usually decided about one year ahead of the meeting, and it reflects the meeting chairs' best judgment of what is most interesting in the field of materials research. The symposium organizers are carefully chosen, too, to provide access to the most interesting and useful subtopics. All of this program-building is done with the assistance and experience of the Program Committee, which is one of MRS's most important working committees, and the MRS staff, who make it all seamless for the member-volunteers. The meeting chairs often add special components of their own, and the upcoming Spring Meeting, for example, has a special session on the role of materials research in the fight against terrorism. Each meeting is different and provides a unique opportunity to learn what is going on in cuttingedge materials research.

The technical symposia are the heart of any MRS meeting, although there are many other activities, too. These include tutorials, a plenary address, a research competition for graduate students, employment exchanges, an equipment exhibit,

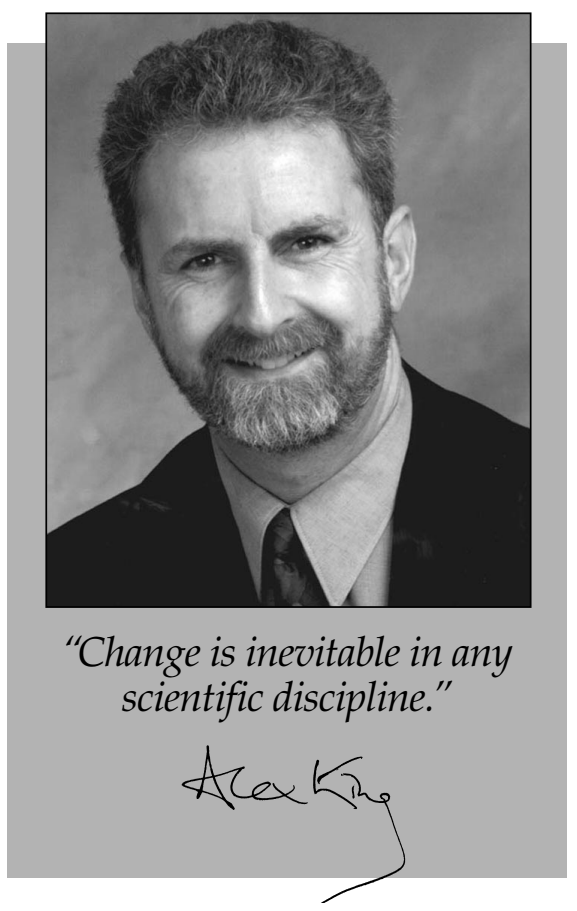

and many others, but the symposium topics are the most clearly defining aspect of any MRS meeting. I have heard MRS criticized for having altered the course of materials science and engineering by its choice of symposium topics, but this does not reflect the true role of the Society. The choice of topics is based upon what is most interesting at any given time, and rather than actually altering the course of our subject, the meetings provide an early indication of its various changes of emphasis and direction. In some cases, this inevitably results in validating a new topic as a subject worthy of study by materials scientists, but that is only a byproduct, not a deliberate agenda. Change is inevitable in any scientific discipline. MRS reflects the changes in materials science, quickly, accurately, and effectively. And, yes, sometimes it is hard to keep up, but you cannot remove that need by denying its existence.

Our lack of "ownership" in any technical area is both an advantage for MRS (allowing the meetings always to be topical and interdisciplinary) and a disadvan- tage (because we tend to provide less programming in maturing areas, and so we sometimes lose the communities associated with them from our membership). MRS has made a conscious decision to limit the size of its Spring and Fall Meetings, keeping the symposia broadly focused and interdisciplinary, and this sometimes forces difficult choices about what topics are included. The decision usually goes in favor of the emerging subjects, and MRS has an enviable record of programming in new areas. Our programs often have conflicts-I frequently have a choice of symposia to which I could submit my own abstracts-but this reflects the interdisciplinary nature of the field and the fact that each of us may be defined by the materials we work on, or their form (e.g., thin film, bulk, quantum dots), or the techniques we use, or the technologies for which they are intended. We strive to avoid overlapping subjects in our programs in the complicated, multidimensional world that we inhabit, and we often create joint sessions to exploit partial overlaps of interest between differing symposium topics. Still, some unplanned overlaps are inevitable and only demonstrate the vitality of the work that is being reported at the meeting, since it will often be of interest to differing communities.

If you want a good overview of what is hot in the areas related to electronic and optoelectronic materials, molecular and biomaterials, or nano-/microstructured materials, come to San Francisco for the MRS Spring Meeting, in the week of April 1-5. You will certainly learn something, you will probably have fun, and you will hopefully contribute something of value. Meeting Chairs Zhenan Bao (Lucent Technologies), Eugene Fitzgerald (Massachusetts Institute of Technology), Ulrich Goesele (Max Planck Institute of Microstructure Physics), and Kenneth Rodbell (IBM T.J. Watson Research Center) have put together a strong program, clearly reflecting the state of our art. I encourage you to come and take advantage of it.

Alex KING 2002 MRS President

\section{MIS Membership Directory}

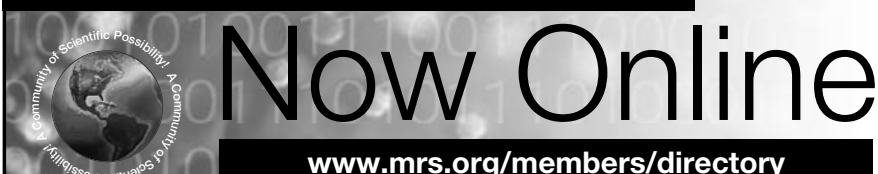

Flexible_search by name, institution, and geographical location

Current_regularly updated

Convenient-linked to the MRS activities and contacts that will help you make the most of your MRS member benefits and services

Exclusive_professional networking service FREE to current MRS members only 